\title{
Nivel de conocimiento de los padres de familia sobre las manifestaciones de depresión en niños de dos centros educativos
}

\author{
De la Cruz-Vila Nery ${ }^{1}$, Flores-Díaz Katia ${ }^{1}$, Salazar-García María del Carmen ${ }^{2}$
}

\section{RESUMEN}

Objetivo: determinar el nivel de conocimiento de los padres de familia sobre las manifestaciones de la depresión en niños. Material y métodos: estudio descriptivo y de corte transversal. La muestra estuvo conformada por 257 padres de familia de niños que estudian en los Centros Educativos Estatales $N^{\circ} 2022$ y N ${ }^{\circ} 3040$ del distrito de Los Olivos. Para la recolección de datos se utilizó un cuestionario elaborado por las investigadoras; éste estuvo dividido en dos partes: 1) datos sociodemográficos del padre/madre; 2) 35 preguntas relacionadas con las manifestaciones emocionales, motoras, cognitivas, conductuales, sociales, psicosomáticas de la depresión en niños; éste fue sometido a juicio de expertos y prueba piloto. Para el análisis de los datos se empleó la estadística descriptiva mediante programa SPSS versión 15.0. Resultados: el 77,8 \% de los padres tenía entre 25 a 44 años de edad, el 87,5\% era de sexo femenino y el 60,3 \% tenía grado de instrucción secundaria. El 85,3\% presentó conocimientos deficientes sobre las manifestaciones generales de la depresión en niños, destacándose en cada área 89,9\%; 79,4\%; 90.7\%;77,4\%; 86,8\%; 76,3\% respectivamente, los padres que alcanzaron conocimiento entre medio y alto fueron aquellos que tenían grado de instrucción secundaria y superior. Conclusiones: los padres de familia presentaron un nivel de conocimiento deficiente sobre las manifestaciones de la depresión en niños.

Palabras clave: padres de familia, conocimiento, niños, manifestaciones de depresión en niños. Perú. (Fuente DeCs BIREME).

\section{Knowledge Level of Parents on the Manifestations of depression in children two Schools}

\begin{abstract}
Objetive: To determine the level of knowledge of the family parents on the manifestations of the depression in children. Material and Methods: : Descriptive study and of transverse court. The sample was shaped by 257 family parents of children who study in the Educational State Centers $\mathrm{N}^{\circ} 2022$ and $\mathrm{N}^{\circ} 3040$ of the district of the Olive trees. For the compilation of information a questionnaire elaborated by the investigators was in use, this one was divided in two parts: 1) information sociodemográficos of the father / mother 2) 35 questions related to the emotional manifestations, motorboats, cognitive, behavioral, social, psycosomatic of the depression in children; this one was submitted in the opinion of experts and pilot test. For the analysis of the information, the descriptive statistics used by means of program SPSS version 15.0. Results: The $77.8 \%$ of the parents had between 25 to 44 years of age, $87.5 \%$ was of feminine sex and $60.3 \%$ had degree of secondary instruction. $85.3 \%$ presented deficient knowledge on the general manifestations of the depression in children, being outlined in every area $89.9 \%, 79.4 \%, 90.7 \%, 77.4 \%, 86.8 \%, 76.3 \%$ and $76.3 \%$ respectively, the parents who reached knowledge between averages and high they were those that had degree of secondary and top instruction. Conclusions. The family parents presented a level of deficient knowledge on the manifestations of the depression in children.
\end{abstract}

Key words: Parents of family, knowledge, children, manifestations of depression in children. Peru. (Source DeCs BIREME). 


\section{INTRODUCCIÓN}

La depresión infantil en los años 70 aún no era considerada como una patología. Entonces se pensaba que los niños eran seres alegres por naturaleza, lloran y están tristes por estados pasajeros (1). En el año 1975, la depresión infantil es aceptada como una patología por el Nacional Institute of Mental Health (Estados Unidos) (2).

La depresión actualmente es la principal causa de suicidios de menores en el Perú. En el año 2009 se reportaron 80 suicidios de menores. Esta alarmante cifra fue obtenida por el Centro para el Control y Prevención de Enfermedades que, además, registró 300 intentos de suicidio de niños en ese período. La tasa de suicidios de menores pasó del 10\% en el 2009, al 12\% en el 2010 (3).

En nuestro país el Ministerio de Salud atiende a un promedio de 600 niños con depresión al año, un tercio de los cuales presenta cuadros severos con ideación suicida e intento de autoeliminación (4). La Dirección Ejecutiva de Investigación y Docencia de Atención Especializada en niños y adolescentes del Instituto Especializado de Salud Mental Honorio Delgado Hideyo Noguchi encontró en el año 2004, que del $15 \%$ de atendidos, la depresión fue la segunda causa más frecuente de consultas en niños y adolescentes con un $10 \%$; en su mayoría procedían del cono norte con un $85,06 \%$ (5).

La depresión en niños se manifiesta por ciertas vivencias subjetivas y conductas observables. En el lado subjetivo destacan fundamentalmente los sentimientos de tristeza y desgano que son los discriminantes básicos de los sujetos que padecen una depresión; en el plano del comportamiento se aprecian conductas de apatía, trastornos somáticos y cansancio (6).

Las manifestaciones de la depresión en niños, según algunos estudios, son detectadas con cierta dificultad, especialmente por los padres de familia quienes subestiman este trastorno afectivo y se resisten a ver los síntomas depresivos en sus hijos o por alguna razón no son capaces de apreciarlos (7).

La enfermería como ciencia y arte, desempeña un papel importante en la prevención de problemas de salud, ya que tiene como centro de atención a la persona, en sus diferentes etapas evolutivas, como un ser integral con aspectos biológicos, psicológicos, sociales y espirituales; en interacción con un medio ambiente que lo rodea y con el cual interactúa. En nuestro medio las enfermeras pueden constituirse en piezas claves de apoyo por encontrarse en los diversos programas y servicios de salud, tanto en instituciones públicas como privadas (8).

Este apoyo, básicamente se orientaría a brindar información, consejería y educación continua sobre las manifestaciones de depresión en niños, a los familiares y miembros de la comunidad, por su sensibilidad, capacidad cognoscitiva y de relaciones interpersonales, además del lenguaje sencillo que suele utilizar cuando se trata de destecnificar los términos clínico-patológicos.

En nuestra práctica comunitaria y clínica se observó el impacto negativo de la falta de conocimiento que tienen los padres de familia, sobre temas relacionados con la salud mental.

De este análisis de la realidad, la escasa literatura identificada a nivel local y nacional, además del déficit de programas educativos y centros especializados en esta área en el Perú, las investigadoras se plantearon el desarrollo de la presente investigación, reconociendo el rol importante que desempeñan los padres de familia en la detección de la depresión en niños; de ahí la importancia de partir haciendo un diagnóstico sobre el nivel de conocimiento que tienen los padres de familia en relación a las manifestaciones de la depresión en niños. Con la finalidad de que la información obtenida pueda ser utilizada por las autoridades del CLAS San Martín y de los centros educativos, donde se realizó el estudio, para que éstos puedan tomar las medidas necesarias ante el problema evidenciado. El objetivo fue determinar el nivel de conocimiento de los padres de familia sobre las manifestaciones de la depresión en niños de los Centros Educativos Estatales $\mathrm{N}^{\mathrm{o}}$ 2022 y No 3040 que pertenecen a la jurisdicción del CLAS San Martin.

\section{MATERIAL Y MÉTODOS}

El estudio de tipo descriptivo y de corte transversal con enfoque cuantitativo, se realizó en dos centros educativos del CLAS San Martín: No 2022 y No 3040 ubicados en el distrito de los Olivos y brindan servicio educativo a nivel primario y secundario.

La población estuvo conformada por 775 padres de familia, la muestra fueron 257 padres de familia, siendo 150 del CEE N³040 y 107 del CEE N²022.

La técnica para la recolección de datos fue la encuesta y el instrumento a utilizar fue el cuestionario autoaplicado, diseñado por las investigadoras según la escala de Likert, fue sometido a juicio de expertos y prueba piloto con 7 
profesionales de la salud.

El cuestionario elaborado constó de dos partes: 1) datos sociodemográficos del padre/madre de familia; y 2) preguntas relacionadas con las manifestaciones de la depresión infantil con 35 ítems.

Las alternativas de respuesta por cada ítem fueron: nunca, a veces y casi siempre; dichos valores se calificaron con 0,1 y 2 . Estos puntajes variaron de acuerdo a la forma en que estaban planteadas las preguntas. Así, cada ítem se puntuaba con un valor de 0 - 2 , la respuesta «nunca» siempre se puntuaba con 0 , y las respuestas «a ve- ces» y «casi siempre» se valoraron con 1 o con 2 según estaban formuladas las preguntas.

Una vez aplicado a los padres de familia el instrumento de recolección de información, se procedió a la asignación de valores a cada una de las respuestas, tomando en cuenta los parámetros establecidos en el instrumento aplicado.

Los cuestionarios se calificaron en forma directa y manual. Se utilizó estadística descriptiva y el programa estadístico SPSS versión 15, se presentan en cuadros de doble entrada.

\section{RESULTADOS}

Tabla 1. Nivel de conocimiento de los padres de familia sobre las manifestaciones de la depresión en niños según el grado de instrucción en los colegios 3040 y 2022 junio, 2011

\begin{tabular}{|c|c|c|c|c|}
\hline \multirow{2}{*}{$\begin{array}{l}\text { Grado de } \\
\text { instrucción }\end{array}$} & \multicolumn{3}{|c|}{ Conocimientos } & \multirow{2}{*}{ Total } \\
\hline & Bajo & Medio & Alto & \\
\hline \multirow{2}{*}{ Primaria } & 44 & 25 & 2 & 71 \\
\hline & $62,0 \%$ & $35,2 \%$ & $2,8 \%$ & $100,0 \%$ \\
\hline \multirow{2}{*}{ Secundaria } & 47 & 82 & 26 & 155 \\
\hline & $30,3 \%$ & $52,9 \%$ & $16,8 \%$ & $100,0 \%$ \\
\hline \multirow{2}{*}{ Superior } & 5 & 16 & 10 & 31 \\
\hline & $16,1 \%$ & $51,6 \%$ & $32,3 \%$ & $100,0 \%$ \\
\hline \multirow{2}{*}{ Total } & 96 & 123 & 38 & 257 \\
\hline & $37,4 \%$ & $47,9 \%$ & $14,8 \%$ & $100,0 \%$ \\
\hline
\end{tabular}

Tabla 2. Nivel de conocimientos de los padres de familia sobre las manifestaciones de la depresión en niños en los colegios 3040 y 2022 junio, 2011

\begin{tabular}{rrrrr}
\hline \multicolumn{1}{c}{ Colegio } & \multicolumn{1}{c}{ Bajo } & \multicolumn{2}{c}{ Conocimientos } \\
Medio & \multicolumn{1}{c}{ Alto } & \multicolumn{1}{c}{ Total } \\
\hline \multirow{2}{*}{ CEE No 3040} & 58 & 65 & 27 & 150 \\
& $38,7 \%$ & $43,3 \%$ & $18,0 \%$ & $100,0 \%$ \\
CEE No 2022 & 38 & 58 & 11 & 107 \\
\multirow{2}{*}{ Total } & $35,5 \%$ & $54,2 \%$ & $10,3 \%$ & $100,0 \%$ \\
& 96 & 123 & 38 & 257 \\
& $37,4 \%$ & $47,9 \%$ & $14,8 \%$ & $100,0 \%$ \\
\hline
\end{tabular}

Tabla 3. Nivel de conocimientos de los padres de familia sobre las manifestaciones emocionales de la depresión en niños en los colegios 3040 y 2022 junio, 2011

\begin{tabular}{lcccc}
\hline \multicolumn{1}{c}{ Colegio } & Bajo & $\begin{array}{c}\text { Emocional } \\
\text { Medio }\end{array}$ & Alto & Total \\
\hline \multirow{2}{*}{$\mathrm{CEE} \mathrm{N}^{\circ} 3040$} & 48 & 81 & 21 & 150 \\
& $32,0 \%$ & $54,0 \%$ & $14,0 \%$ & $100,0 \%$ \\
CEE No 2022 & 33 & 69 & 5 & 107 \\
\multirow{2}{*}{ Total } & $30,8 \%$ & $64,5 \%$ & $4,7 \%$ & $100,0 \%$ \\
& 81 & 150 & 26 & 257 \\
& $31,5 \%$ & $58,4 \%$ & $10,1 \%$ & $100,0 \%$ \\
\hline
\end{tabular}


Tabla 4. Nivel de conocimientos de los padres de familia sobre manifestaciones motoras de la depresión en niños en los colegios 3040 y 2022 junio, 2011

\begin{tabular}{lcccc}
\hline \multirow{2}{*}{ Colegio } & Bajo & $\begin{array}{c}\text { Motoras } \\
\text { Medio }\end{array}$ & Alto & Total \\
\hline \multirow{2}{*}{$\mathrm{CEE} \mathrm{N}^{\circ} 3040$} & 29 & 88 & 33 & 150 \\
& $19,3 \%$ & $58,7 \%$ & $22,0 \%$ & $100,0 \%$ \\
CEE N 2022 & 15 & 72 & 20 & 107 \\
& $14,0 \%$ & $67,3 \%$ & $18,7 \%$ & $100,0 \%$ \\
Total & 44 & 160 & 53 & 257 \\
& $17,1 \%$ & $62,3 \%$ & $20,6 \%$ & $100,0 \%$ \\
\hline
\end{tabular}

Tabla 5. nivel de conocimientos de los padres de familia sobre manifestaciones cognitivas de la depresión en niños en los colegios 3040 y 2022 junio, 2011

\begin{tabular}{lcccc}
\hline Colegio & Bajo & $\begin{array}{c}\text { Cognitiva } \\
\text { Medio }\end{array}$ & Alto & Total \\
\hline \multirow{2}{*}{ CEE N 3040} & 43 & 91 & 16 & 150 \\
& $28,7 \%$ & $60,7 \%$ & $10,7 \%$ & $100,0 \%$ \\
$\mathrm{CEE} \mathrm{N}^{\circ} 2022$ & 37 & 62 & 8 & 107 \\
& $34,6 \%$ & $57,9 \%$ & $7,5 \%$ & $100,0 \%$ \\
Total & 80 & 153 & 24 & 257 \\
& $31,1 \%$ & $59,5 \%$ & $9,3 \%$ & $100,0 \%$ \\
\hline
\end{tabular}

Tabla 6. Nivel de conocimientos de los padres de familia sobre manifestaciones sociales de la depresión en niños en los colegios 3040 y 2022 junio, 2011

\begin{tabular}{lcccc}
\hline \multirow{2}{*}{ Colegio } & Bajo & $\begin{array}{c}\text { Sociales } \\
\text { Medio }\end{array}$ & Alto & Total \\
\hline \multirow{2}{*}{$\mathrm{CEE} \mathrm{N} 3040$} & 57 & 58 & 35 & 150 \\
& $38,0 \%$ & $38,7 \%$ & $23,3 \%$ & $100,0 \%$ \\
$\mathrm{CEE} \mathrm{N}^{\circ} 2022$ & 39 & 45 & 23 & 107 \\
& $36,4 \%$ & $42,1 \%$ & $21,5 \%$ & $100,0 \%$ \\
Total & 96 & 103 & 58 & 257 \\
& $37,4 \%$ & $40,1 \%$ & $22,6 \%$ & $100,0 \%$ \\
\hline
\end{tabular}


Tabla 7. Nivel de conocimientos de los padres de familia sobre manifestaciones conductuales de la depresión en niños en los colegios 3040 y 2022 junio, 2011

\begin{tabular}{lcccc}
\hline \multirow{2}{*}{ Colegio } & Bajo & $\begin{array}{c}\text { Conductuales } \\
\text { Medio }\end{array}$ & Alto & Total \\
\hline \multirow{2}{*}{ CEE No 3040} & 49 & 83 & 18 & 150 \\
& $32,7 \%$ & $55,3 \%$ & $12,0 \%$ & $100,0 \%$ \\
CEE No 2022 & 41 & 50 & 16 & 107 \\
& $38,3 \%$ & $46,7 \%$ & $15,0 \%$ & $100,0 \%$ \\
\multirow{2}{*}{ Total } & 90 & 133 & 34 & 257 \\
& $35,0 \%$ & $51,8 \%$ & $13,2 \%$ & $100,0 \%$ \\
\hline
\end{tabular}

Tabla 8. Nivel de conocimientos de los padres de familia sobre manifestaciones psicosomáticas de la depresión en niños en los colegios 3040 y 2022 junio, 2011

\begin{tabular}{lcccc}
\hline Colegio & Bajo & $\begin{array}{c}\text { Psicosomáticas } \\
\text { Medio }\end{array}$ & Alto & Total \\
\hline \multirow{2}{*}{ CEE N 3040} & 42 & 75 & 33 & 150 \\
& $28,0 \%$ & $50,0 \%$ & $22,0 \%$ & $100,0 \%$ \\
CEE N 2022 & 26 & 53 & 28 & 107 \\
& $24,3 \%$ & $49,5 \%$ & $26,2 \%$ & $100,0 \%$ \\
Total & 68 & 128 & 61 & 257 \\
& $26,5 \%$ & $49,8 \%$ & $23,7 \%$ & $100,0 \%$ \\
\hline
\end{tabular}

En la Tabla 1, el nivel de conocimientos sobre manifestaciones de la depresión en niños fue alto en un $32,3 \%$ de los padres con nivel de instrucción superior y sólo el 2,8\% de los padres con nivel de instrucción primaria tenía conocimiento alto. Las diferencias encontradas resultaron estadísticamente significativas $(\mathrm{p}=0,00)$.

En la Tabla 2, el nivel de conocimientos de los padres de familia sobre las manifestaciones de depresión en niños que predominó, fue el medio con un 47,9\% y sólo el 14,8\% mostró nivel de conocimientos alto.

En ambos colegios predominó el nivel de conocimientos medio, con $43,3 \%$ para el CEE N 3040 y de $54,2 \%$ para el CEE N $\mathrm{N}^{\mathrm{2}} 2022$. No se presentaron diferencias significativas $(\mathrm{p}=0,121)$.

En la Tabla 3, el nivel de conocimiento de los padres de familia sobre manifestaciones emocionales de depresión en niños que predominó, fue el medio con un $58,4 \%$ y sólo el 10,1\% mostró nivel de conocimientos alto.

En ambos colegios predominó el nivel de conocimiento medio, sin embargo para el CEE No 3040 el porcentaje con nivel de conocimientos alto fue del $14,0 \%$ mientras que para el CEE $\mathrm{N}^{\circ} 2022$ este porcentaje fue del 4,7\%, siendo la diferencia estadísticamente significativa. $(p=0,037)$

En la Tabla 4, el nivel de conocimiento de los padres de familia sobre las manifestaciones motoras de depresión en niños que predominó, fue el medio con un $62,3 \%$ y sólo $20,6 \%$ de padres de familia presentó conocimiento alto.

En ambos colegios predominó el nivel de conocimientos medio, con $58,7 \%$ para el CEE No 3040 y $67,3 \%$ para el $\mathrm{CEE} \mathrm{N}^{\mathrm{o}} 2022$. No se presentaron diferencias significati$\operatorname{vas}(p=0,349)$.

En la Tabla 5, el nivel de conocimiento de los padres de familia sobre las manifestaciones cognitivas de la depresión en niños que predominó, fue el medio con un 59,5\% y sólo el 9,3\% mostró nivel de conocimientos alto.

En ambos colegios predominó el nivel de conocimiento medio, sin embargo para el CEE No 3040 el porcentaje con nivel de conocimientos bajo fue del $28,7 \%$ mientras 
que para el CEE $\mathrm{N}^{\circ} 2022$ este porcentaje fue del 34,6\%, siendo la diferencia estadísticamente significativa. $(\mathrm{p}=0,482)$

Observamos en la Tabla 6 el nivel de conocimiento de los padres de familia sobre las manifestaciones sociales de depresión en niños que predominó, fue el medio con un $40,1 \%$ y sólo el $22,6 \%$ mostró nivel de conocimientos alto.

En ambos colegios predominó el nivel de conocimientos medio, con $38,7 \%$ para el CEE No 3040 y de $42,1 \%$ para el CEE $\mathrm{N}^{\circ} 2022$. No se presentaron diferencias significativas $(\mathrm{p}=0,855)$.

En la Tabla 7, el nivel de conocimiento de los padres de familia sobre las manifestaciones conductuales de depresión en niños que predominó, fue el medio con un 51,8\% y sólo el 13,2\% mostró nivel de conocimientos alto.

En ambos colegios predominó el nivel de conocimientos medio, con 55,3\% para el CEE No 3040 y $46,7 \%$ para el $\mathrm{CEE} \mathrm{N}^{\mathrm{o}} 2022$. No se presentaron diferencias significati$\operatorname{vas}(p=0,392)$.

Podemos obsevar en la Tabla 8 el nivel de conocimiento de los padres de familia sobre las manifestaciones psicosomáticas de depresión en niños que predominó, fue el medio con un $49,8 \%$ y sólo el $23,7 \%$ mostró nivel de conocimiento alto.

En ambos colegios predominó el nivel de conocimiento medio, sin embargo para el CEE $\mathrm{N}^{\mathrm{o}} 2022$ el porcentaje con nivel de conocimientos alto fue del $26,2 \%$ mientras que para el CEE No 3040 este porcentaje fue del 22,0\%, siendo la diferencia estadísticamente significativa. $(\mathrm{p}=0,676)$.

\section{DISCUSIÓN}

Morales L. (2007), en su estudio «Detección de síntomas depresivos en niñas y niños» refiere que en ocasiones, observamos a niñas y niños que por diferentes razones se aíslan, están tristes e introvertidos, a veces también, inquietos e irritables, conductas no siempre valoradas en forma adecuada por algunos adultos, quienes al convivir con el infante pueden optar por marginarlo o etiquetarlo como «niño problema», pues no logran explicarse qué sucede con el menor. Sin embargo, al ahondar más en la historia de vida del niño, aparecen otros datos importantes, tales como: cambios en los hábitos de sueño, de ape- tito, distracciones frecuentes, fatiga y molestias vagas sin causas fundamentadas (9).

El presente estudio tuvo como objetivo determinar el nivel de conocimiento de padres de familia sobre las manifestaciones de depresión en niños, teniendo en cuenta las áreas: emocionales, motoras, conductuales, cognitivas, sociales y psicosomáticas, que pueden ser afectadas por este trastorno.

Con respecto al nivel de conocimiento de los padres de familia en relación al grado de instrucción, los resultados evidencian que los padres que alcanzaron un nivel de conocimiento alto tienen grado de instrucción superior (Tabla 1).

En el estudio realizado por Emma Soto A. (2009) «Nivel de conocimientos de los padres de familia acerca de la salud mental de sus hijos en un centro educativo de Lima», los padres de familia que presentaron un nivel de conocimiento entre medio y alto fueron en su mayoría de grado de instrucción superior y secundaria completa (10). Asi mismo, en una investigación ejecutada por el Dr. Gutierrez, Carlos (2001), Percepción de la Patología Mental en Lima Metropolitana, aplicada a una muestra de personas de nivel cultural aceptable y que no eran profesionales ni técnicos en salud, se pudo apreciar que en general no poseen conocimientos sólidos sobre depresión; se encontró que el $65,5 \%$ de la muestra, poseía conocimientos entre medio y bajo.

La Organización Panamericana de la Salud, en cuanto al grado de instrucción, refiere que todo individuo al nacer posee un potencial determinado de capacidades. El desarrollo de éste está relacionado con el grado de instrucción alcanzado, lo que a su vez afecta o repercute directamente en su estado de salud. Es decir, a mayor grado de instrucción, habrá mejor comprensión (12). En este sentido, se destaca la educación de los padres de familia como un aspecto esencial para asegurar la promoción de la calidad de vida y futuro de sus hijos.

Esto nos lleva a definir que el grado de instrucción influye en el nivel de conocimiento ya que la educación da mayor capacidad de análisis y de crítica.

En relación al nivel de conocimiento de los padres de familia sobre las manifestaciones de la depresión en niños, se evidenció que del total de 257 padres de familia que participaron en la investigación, el 47,9\% alcanzó un nivel de conocimiento medio, seguido del bajo con un $37,4 \%$ y sólo el $14,8 \%$ presentó un nivel alto de conocimiento. Es 
decir que el 85,2\% de padres de familia posee un conocimiento de medio a bajo sobre las manifestaciones de depresión en niños (Tabla 2).

Estos resultados pueden estar relacionados con diversos factores como: la escasez de programas educativos estructurados sobre salud mental (depresión en niños), actividades laborales de los padres de familia que pueden interferir en su participación en los programas educativos, deficiente información y falta de difusión de los medios de comunicación sobre el tema expuesto.

La familia es el entorno más inmediato del niño, en sus cuidados y atención se basa la posibilidad de supervivencia del niño. El modelo de sociedad actual ha generado que los niños pasen cada vez más tiempo solo. Los padres pasan gran parte del tiempo tratando de ganar dinero y de mantener la vida social que creen necesaria, pero dedican pocas horas al niño y la soledad les lleva a la depresión. El niño deprimido va a manifestar su malestar creando problemas para que se le preste atención. Se muestra triste, aburrido, irritable, retraído, tiende a la soledad o a la rebeldía (13). Si estas manifestaciones son reconocidas por parte de los padres como parte de este trastorno afectivo, no pasarían por desapercibidas, sino por el contrario se tomarían las medidas necesarias evitando así que éstas puedan limitar las posibilidades futuras del niño ya que pueden truncar su desarrollo e incluso llevarlo al suicidio. Dar solución a este problema ahorraría tiempo, dinero y naturalmente sufrimiento en el niño.

Algunos niños llegan a suicidarse. No todos los niños deprimidos son adultos deprimidos, pero casi todos los adultos deprimidos han sido niños deprimidos.

Becerra, Cribillero y Flores (1998) realizaron en nuestro país, un estudio que muestra la existencia de déficit de conocimientos en los familiares de pacientes deprimidos en cuanto a sintomatología, diagnóstico, etiología, administración de medicamentos, manejo del paciente y tratamiento (14).

En relación al nivel de conocimiento de los padres de familia sobre las manifestaciones emocionales de la depresión en niños, se evidenció que el 58,4\% alcanzó un nivel de conocimiento medio, seguido del $31,5 \%$ con un conocimiento bajo y sólo el 10,1\% con un conocimiento alto; es decir que el $89 \%$ presenta un conocimiento de regular a deficiente, concluyendo que la mayoría de los padres de familia no tienen conocimientos sólidos sobre las manifestaciones emocionales de la depresión en el niño (Tabla 3).
Según Méndez (2007), la infelicidad y tristeza prolongada sólo la puede sentir el que padece este trastorno afectivo, pero las manifestaciones emocionales de la depresión tales como la facies de tristeza, llanto con facilidad, irritabilidad, ausencia de interés por las cosas, las ven y escuchan las personas que conviven a su alrededor. Los padres de familia pueden observar estas manifestaciones emocionales en las personas con quienes interactúan, especialmente en sus hijos, pero al no saber suficientemente que éstas son manifestaciones emocionales de la depresión, tienden a minimizar estos signos de alarma sin tomar ninguna medida ante esto (15).

En relación al nivel de conocimiento de los padres de familia sobre las manifestaciones motoras de la depresión en niños, se evidenció que el $62,3 \%$ alcanzó un nivel de conocimiento medio, seguido del $20,6 \%$ con un conocimiento alto y solo el $17,1 \%$ con un conocimiento bajo; es decir que el 79,4\% presenta un nivel de conocimiento de medio a bajo, concluyendo que la mayoría de los padres de familia no tienen conocimientos sólidos sobre las manifestaciones motoras de la depresión en el niño. (Tabla 4).

La depresión es un problema complejo que incluye alteraciones motoras como la agitación o enlentecimiento psicomotor, que se manifiestan por el paso de una actividad a otra sin terminar la anterior, realizar actividades diarias con letargo, pérdida de energía y cansancio (16). Estos signos, al no ser reconocidos por los padres como manifestaciones de la depresión, muchas veces los llevan a hacer juicios negativos, considerando al niño como «holgazán», «malcriado», «ocioso», «flojo». Muchas veces no solo hay maltrato verbal sino también físico ocasionando así en el niño mayor depresión. Por ello la necesidad urgente de que los padres puedan conocer estas manifestaciones motoras de depresión en el niño, ya que en la prevención de este trastorno ellos cumplen un papel preponderante, pero llevarán a cabo esta tarea sólo cuando estén bien informados.

En relación al nivel de conocimiento de los padres de familia sobre las manifestaciones cognitivas de la depresión en niños, se evidenció que el 59,5\% alcanzó un nivel de conocimiento medio, seguido del $31,2 \%$ con un conocimiento bajo y sólo el 9,3\% con un conocimiento alto; es decir que el 90,7\% presenta un nivel de conocimiento de medio a bajo (Tabla 5).

J. Royo en su artículo «Trastornos depresivos en la infancia y adolescencia» refiere que conforme el nivel de desarrollo cognitivo va progresando, el niño con depresión puede ser capaz de mostrar componentes cognitivos de 
su humor disfórico y baja autoestima, así como sentimientos de culpa y de infelicidad (17).

Batista (2006) en su estudio «Síntomas de depresión, atención sostenida y desempeño escolar en estudiantes», concluyó que la depresión incluye síntomas cognitivos diversos tales como problemas de atención, que a su vez pueden perjudicar el desempeño escolar (18). Asimismo, Rodríguez. R. en su estudio «El síndrome depresivo en el niño y en el adolescente», refiere que en los niños escolares los síntomas más importantes se van a referir a la esfera cognitiva, manifestándose a través de bajo rendimiento escolar, rumiación de ideas, sentimientos de inferioridad e impulsos suicidas (19).

Teniendo en cuenta la referencia de los estudios hallados y los resultados del nivel de conocimiento deficiente que alcanzaron los padres de familia sobre las manifestaciones cognitivas de la depresión, se requiere, por su gran importancia, que éstos puedan conocer especialmente si el descenso brusco del rendimiento escolar del niño puede estar relacionado con la depresión, ya que como refiere B. Bulnes, el fracaso escolar puede ser una causa o efecto más relevante de la depresión (20). Asimismo se observa que por diversas razones, los padres no hacen seguimiento del avance escolar de sus hijos, de igual manera no participan de las reuniones convocadas por los maestros en las instituciones educativas.

En la Tabla 6, en relación al nivel de conocimiento sobre las manifestaciones sociales de la depresión, se aprecia que el $40,0 \%$ de los padres de familia tiene un nivel de conocimiento medio, seguido del $37,4 \%$ de padres de familia con un conocimiento bajo; es decir que el $77,4 \%$ de los padres de familia posee un nivel de conocimiento de medio a bajo.

La infancia intermedia, que viene a ser la etapa escolar, se caracteriza por la gran capacidad de socialización y formación de grupos, en la que los niños crean su propia cultura de secretos, costumbres, etc. con los cuales fomentan los sentimientos de solidaridad grupal (21).

Las manifestaciones sociales que caracterizan a un niño con depresión son la menor participación en el grupo, menor interés social y es probable que no intenten hacer amigos. Considerando que durante la etapa escolar la socialización se da en grado eminente, los signos de aislamiento en los niños deben ser reconocidos por los padres de familia como signo de que algo no anda bien en el niño. Cabe resaltar también, que los padres podrán reconocer estas manifestaciones siempre y cuando conozcan las carac- terísticas normales de socialización en el niño de 7 a 12 años de edad.

Ajuriagerra, J. en su libro «Manual de Psiquiatría Infantil», refiere que los escolares deprimidos exhiben los problemas más de tipo conductuales, que permiten su identificación y descripción (22).

En la Tabla 7, respecto al conocimiento de las manifestaciones conductuales de la depresión, se aprecia que el $51,8 \%$ de los padres de familia tiene un nivel de conocimiento medio, seguido del $35,0 \%$ de padres de familia con un conocimiento bajo; es decir que el $86,8 \%$ de los padres de familia tiene un conocimiento deficiente y sólo el 13,2\% posee un nivel de conocimiento alto.

Esto indica que en nuestra población de estudio aún no se consolida la idea de que el comportamiento de agresividad, labilidad emocional, rabietas, protestas ante las indicaciones, pueden ser manifestaciones conductuales de depresión si se presentan por tiempo prolongado. Pues muchas veces estos comportamientos son considerados por los padres como normales para la edad y no les dan la debida importancia.

En la Tabla 8 se muestra los hallazgos del nivel de conocimientos de los padres de familia, en relación a las manifestaciones psicosomáticas de la depresión. El nivel de conocimiento que predominó fue el medio con un $49.8 \%$, seguido del bajo con un $26,5 \%$. Es decir, el $76,3 \%$ de padres de familia posee conocimientos entre medio a bajo y el 23,7 alcanzó un nivel de conocimiento alto.

Las molestias físicas sin explicación médica corresponden a los denominados trastornos de somatización, llamados también depresión enmascarada, donde el escolar trasforma un problema psicológico en un síntoma físico; se obsesiona con disfunciones corporales como cefalea, dolor abdominal, pérdida o aumento de sueño y apetito (23). Rodríguez, R. refiere que los síntomas físicos, como las quejas somáticas, son particularmente frecuentes en niños con depresión, siendo la cefalea un síntoma dominante en el escolar (19).

Rivera P. refiere que numerosos niños tienen dificultades para expresar el sufrimiento psíquico y lo hacen en forma de quejas somáticas, de manera que obstaculizan el reconocimiento de trastornos psiquiátricos y, sin embargo, padecen cuadros depresivos primarios denominados tradicionalmente depresión enmascarada (24).

Como refiere Rivera, existe dificultad para poder recono- 
cer los trastornos psiquiátricos cuando de por medio están la quejas somáticas, pues se cree que éstas sólo tienen origen físico. Por ello es importante que los padres de familia puedan conocer que la depresión en el niño también tiene manifestaciones somáticas.

Estos datos de déficit de conocimiento sobre manifestaciones emocionales, conductuales, cognitivas, psicosomáticas, sociales y motoras de depresión en los niños, diagnosticados en la evaluación a los padres de familia de los centros educativos donde se realizó el estudio, reflejan la realidad en la que se pueden encontrar muchos de ellos, ya que es común ver campañas de salud concientizando sobre la responsabilidad individual, así como medios difundiendo información sobre la prevención de enfermedades que puedan amenazar la salud, pero centrados en la salud física. Son escasos y desconocidos los que se refieren a la salud mental, de ahí el reto de hacer llegar la atención necesaria a los menores de edad incluyendo una mejor campaña educativa para padres de familia y maestros, con el fin de que éstos puedan tener conocimientos claros y precisos sobre las manifestaciones de la depresión infantil.

El hecho de que los integrantes de una familia, especialmente los padres, se encuentren informados sobre las manifestaciones de la depresión en niños, facilita y favorece mucho que éstas sean detectadas oportunamente ya que, como refiere Flores en su libro Hacia una Pedagogía de la Educación, la acumulación de información eleva la calidad de vida y proporciona herramientas para afrontar con mayor eficacia los problemas que se les presente. No conocer la enfermedad pone en riesgo al individuo y la familia, frente a la detección oportuna y afronte necesario para vencer la enfermedad (25).

\section{REFERENCIAS BIBLIOGRÁFICAS}

1. Méndez Francis X. El Niño que no sonríe. $1^{\mathrm{a}}$ ed. Madrid: Pirámide; 1998. pp. 46- 82.

2. Organización Mundial de la Salud. Promoción de la Salud Mental. Panamá; 2004. pp. 13 -16

3. Diario el Trome. Alarma por suicidio de menores lunes 24 de enero 2011 (citado 18 Set 2011). Disponible en: http://trome.pe/actualidad/703148/noticia-alarmasuicidios-menores.

4. Agencia Peruana de Noticias Andina. Intentos de suicidio infantil se duplicaron en los últimos tres años. [Internet] (citado 28 May 2010). Disponible en: http:/ /www.andina.com.pe/Espanol/
5. Instituto Especializado de Salud Mental Honorio Delgado Hideyo Noguchi. Dirección Ejecutiva de Investigación y Docencia de Atención Especializada. Niños y Adolescentes. Perú; OEA. 2004.

6. Gonzales R. Psicología del niño y del adolescente. ed. Pirámide Madrid; 1998. S.A.

7. Del Barrio V. La depresión infantil, factores de riesgo y posibles soluciones ed. Aljibe Málaga-España; 2000. pp. 17.

8. Ramón J. Enfermería en Atención Primaria. $1^{\circ}$ ed. DAE Madrid-Valencia; 2006. pp. 193- 213.

9. Morales L. Detección de síntomas de depresión en niñas y niños maternales. Universidad del Valle de México: Rectoría Institucional. Episteme No. 10. Año 3, julio-septiembre 2007. Episteme revista académica. Disponible en: http://www.uvmnet.edu/investigacion/ episteme/numero1007/reportes/a_detecSR.asp.

10. Emma Soto. 2010. Anivel de conocimiento de padres de familia acerca de la salud mental de sus hijos preescolares en la Institución Educativa 6084 VMT 2009. Tesis para optar por el título de Licenciada en Enfermería UNMS.

11. Gutiérrez C. Percepción de la Patología Mental en Lima Metropolitana 2001 Tesis para optar el Título de Médico. UNMS.

12. Organización Panamericana de la Salud. Las condiciones de salud en las Américas. Washington. D.C: OPS; 2004.

13. Carmen M. Disponible: conociendo la depresión. 2010. [Internet] (citado 19 Set 2011). Disponible en: http://www.herreros.com.ar/melanco/carmen.htm.

14. Becerra Cribillero, Flores. 1998. Nivel de conocimientos en Familiares de Pacientes con Depresión en el Instituto Nacional de Salud Mental Honorio Delgado Hideyo Noguchi. (tesis). Perú; Universidad Peruana Cayetano Heredia; 1998.

15. Méndez F. El niño que no sonríe. $2^{\circ}$ ed. Pirámide Madrid- España; 2007. pp. 123.

16. Méndez F. El niño que no sonríe. $1^{a}$ Edición Pirámide Madrid- España; 1998. pp. 38-41

17. Royo J. Trastornos depresivos en la niñez y la adolescencia. [Internet] 1 de junio de 2011 (citado 23 Agos 2011) Disponible en: http://www.res puestasaladepresion.com/profesionales/lecturas recomendadas.php\#infanciayadolescencia.

18. Baptista M, Lima R, Capovilla A, Melo L. Sintomatología depresiva, atención sustentada y desempeño escolar en estudiantes [Internet]. 2006, vol.10, n.1 [citado el 23 de agosto del 2011], pp. 99108. Disponible en: http://www.scielo.br/ scielo.php?script $=$ sci_arttext\&pid $=\mathrm{S} 14138557200$ $6000100009 \& \operatorname{lng}=$ en\&nrm=iso $>$. 
19. Rodríguez R. El síndrome depresivo en el niño y en el adolescente. [Internet]. 1999 (citado 23 de agosto); 1(1): p 83-88. Disponibleen:http://www.scielo.sa.cr/ scielo.php? script $=$ sci_arttext\&pid $=$ S 1409 41851999000100013\&lng=es.

20. Bulnes B. Estados depresivos y atmósfera de familiar en niños con bajo rendimiento escolar. Revista de Investigación en Psicología 1998. Vol, No 2, pp.99

- 111. Disponible en: http://sisbib.unmsm.edu.pe/ bvrevistas/investigacion_psicologia/v01_n2/pdf/ a04v1n2.pdf.

21. Wong D. Enfermería Pediátrica. ed. Mosby Doyma. Madrid-España; 1995.pp. 339.

22. Ajuriagerra J. Manual de Psiquiatría Infantil. Barcelona. 1990; Masson. S.A. pp. 2.

23. Ministerio de Salud del Perú. Guía Práctica Clínica: Epidemiología Depresiva. [Internet] Lima-MINSA: 2006 (citado 20 May 2011) disponible en: http:// www.minsa.gob.pe/portal/pe2005/documentos/ Smental/guia\%20DE\%20DEPRESION.doc.
24. Rivera P. Depresión y enfermedades psicosomáticas. España; 2009. pp. 1 Disponible en: http://www. elsevier.es/sites/default/files/elsevier/pdf/40/ 40v35nSupl.1a13190895pdf001.pdf

25. Flores R. Hacia una Pedagogía del Conocimiento. Editorial Mc. Graw-Hill. Colombia; 1994. pp. 97.

\section{Correspondencia}

Maria del Carmen Salazar García

Universidad Peruana Cayetano Heredia

Dirección: Av. Honorio Delgado 430, Urb. Ingeniería, Lima - 31

Correo electrónico: maria.del.carmen.salazar@upch.pe

Forma de citar este artículo: De La Cruz-Vila N, Flores-Díaz K, Salazar-García M. Nivel de Conocimiento de los Padres de familia sobre las Manifestaciones de depresión en niños de dos Centros Educativos. Rev enferm Herediana. 2014;7(2):97-106. 